\title{
Improving Post-Stroke Outcomes in Ischemic Stroke Animal Models Using C3a Monoclonal Antibody Treatment \\ David Failinger ${ }^{1}$, Jui-Hung Yen ${ }^{2}$ \\ ${ }^{1}$ Indiana University School of Medicine, ${ }^{2}$ Department of Microbiology and Immunology, Indiana University School of Medicine
}

\section{Background}

Stroke is one of the leading causes of death in the world and a majority of strokes cases are ischemic stroke. Ischemia causes tissue damage by preventing nutrient delivery, but damage is also caused by inflammation after reperfusion. The complement pathway is active during reperfusion. Our study focuses on the effects of C3a component inhibition during post stroke inflammation.

\section{Material and Methods}

A total of 30 C57BL/6 mice will be randomly divided into 3 groups ( $n=10 / g$ roup) and subjected to a 40-minute middle cerebral artery occlusion (MCAO). The control group will receive a vehicle (saline, Group 1) and two experiment groups will receive $5 \mathrm{ml} / \mathrm{kg}$ IV injections of neutralizing C3a monoclonal antibody. The first experimental group (Group 2) will receive the injection 30 minutes after the start of occlusion and the second experimental group (Group 3) will receive two injections at 30 minutes and $3 \mathrm{~h}$ after the start of occlusion. All groups will be subject to neurological function tests at $24 \mathrm{~h}$ and $48 \mathrm{~h}$. Additionally, at $48 \mathrm{~h}$ post-injury animals will be sacrificed and the ischemic brains harvested, followed by Triphenyl tetrazolium Chloride (TTC) staining to determine infarct volume.

\section{$\underline{\text { Results }}$}

The infarct volumes and neurological function scores for the experimental groups are expected to have statistically smaller values and improved neurological deficient compared to the vehicle group. Group 3 mice are expected to have smaller values and lower scores compared to Group 2.

\section{Discussion}

C3a plays an important role in recruitment of inflammatory cells such as macrophages/microglia and neutrophils, which can cause brain tissue injury and neuron cell death. Limiting the presence C3a component by monoclonal antibody is expected to limit recruitment and lessen post-ischemic inflammation damage. Future studies may test combinations of complement pathway inhibition to optimize the depression of post-stroke inflammation and as well as preventing susceptibility to post-stroke infection. 